\title{
Fast Matched Field Processing
}

\author{
S. Aravindan, N. Ramachandran, and Prabhakar S. Naidu
}

\begin{abstract}
Source localization by matched field processing (MFP) requires computation of theoretical field over a dense grid of source range and depth in the region where the source is likely to be present. The computed field at each grid point is then correlated with the observed field. In this paper we show how the computational burden can significantly be reduced ( 20 to 30 times) by expressing the correlation in terms of discrete Fourier transform and using the fast Fourier transform (FFT) algorithm. A price that one pays to achieve increased speed is in the form of quantization phase errors. It is shown through analysis and computer simulation that the quantization errors reduce the source peak height, depending upon the size of DFT. The proposed fast MFP works for range localization only. However, the depth estimation is possible by repeated application of above algorithm for different depths.
\end{abstract}

\section{INTRODUCTION}

A MATCHED field processor (MFP) estimates the source location by correlating the acoustic pressure received by an array with the replica field, derived from the knowledge of the environment, corresponding to a particular trial source location. The unique spatial structure of the field permits the localization of the source in range, depth, and bearing.

MFP was proposed by Bucker [1] and later developed by Fizell [2] and Baggeroer et al. [3] for different methods. The performance of the MFP with respect to the resolution of closely spaced sources was reported by Ozard [4]. Hamson et al. [5] have reported extensive simulation results on the effect of system parameters like array length, sensor spacing, etc., and environmental parameters like water depth, sound-speed profile, and frequency, etc., on the MFP. Analytical results for the sensitivity of the MFP to mismatch in system parameters and environmental parameters were given by Gingras [6].

Though a considerable amount of work has been done to study the performance of MFP in different environmental conditions, the problem of developing a fast and efficient algorithm to implement MFP has not been studied. Note that source localization using MFP requires $N_{x} \times N_{z}$ searches in the parameter space, where $N_{r}$ and $N_{z}$ are the number of search points in range and depth, respectively. In this paper, we present a fast algorithm based on the fast Fourier transform (FFT) to implement the MFP. We consider an inhomogeneous shallow water channel as shown in Fig. 1. A vertical array of sensors (Fig. 1(a)) or a horizontal array of sensors (Fig. $1(b))$ is used for source localization. In the proposed algorithm each trial source location is not processed separately; instead, a group of trial source locations are processed together at once. This proposed algorithm can be used for the maximum

Manuscript received January 10, 1991; revised June 11, 1992.

The authors are with the Department of Electrical and Communications Engineering, Indian Institute of Science, Bangalore-560 012, India. IEEE Log Number 9203955.

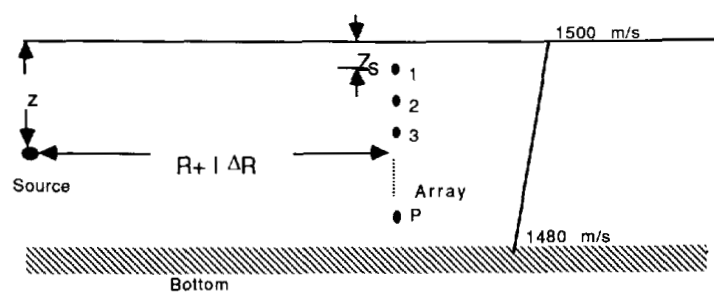

(a)

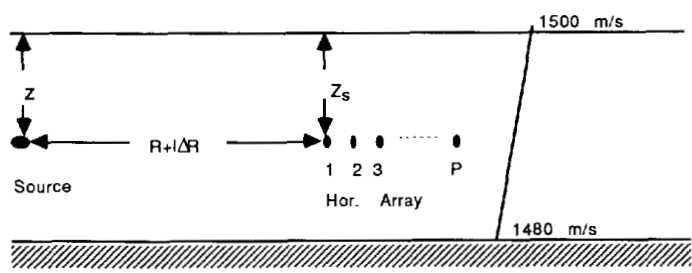

(b)

Fig. 1. Shallow water channel and an array of sensors. Channel character istics: depth $=100 \mathrm{~m}$, linear sound speed variation as shown, hardbottom. Array: 25 sensors equispaced at $3 \mathrm{~m}$. Vertical array is located at $6 \mathrm{~m}$ from the surface. Narrow-band source emitting at $170 \mathrm{~Hz}$. (a) Vertical array. (b) Horizontal array.

likelihood (ML) processor [7] as well as for the Bartlett (Linear) processor. But, here only the ML processor is considered. Computational speed of the order of $20-30$ is predicted. This has been verified through numerical simulations. (Our attention was drawn by one of the referees to a paper on the use of an artificial neural network that speeds MFP, as claimed, by several orders of magnitude [8].)

The paper is organized as follows. A brief summary of ML processor is given in Section II. Our algorithm based on FFT is described in Section III. The theoretical analysis of processing speed enhancement supported by numerical simulation results are shown in Section IV. In the last section a discussion on quantization errors is included.

\section{Maximum LiKelihood Method}

The ML method is a high-resolution array processing method that adaptively constructs the weighting vector consistent with the array geometry and the structure of the field across the array. The weighting vector $\boldsymbol{W}$ is chosen such that any signal from the trial location $(R+1 \Delta R, Z)$ is passed with no distortion while the signals from other locations are regarded as interference and suppressed in an optimal manner. Mathematically, the following function has to be minimized

$$
\mathbf{W}^{H} \mathbf{C W}=\min
$$


subject to the constraint

$$
\boldsymbol{W}^{H} \boldsymbol{P}(R+1 \Delta R, Z)=1
$$

where $C$ is the spectral matrix of the received field vector and $\boldsymbol{P}(R+1 \Delta R, Z)$ is the normalized replica field vector and $H$ stands for Hermitian transpose. The solution to this optimization problem is obtained using the Lagrange multiplier method [3] and is given by

$$
\boldsymbol{W}=\frac{\boldsymbol{C}^{-1} \boldsymbol{P}(R+1 \Delta R, Z)}{\boldsymbol{P}^{H}(R+1 \Delta R, Z) \boldsymbol{C}^{-1} \boldsymbol{P}(R+1 \Delta R, Z)} .
$$

The output of the processor for a trial location $(R+1 \Delta R, Z)$ is given by

$$
\begin{aligned}
S_{\mathrm{ML}}(R+1 & \Delta R, Z) \\
= & \frac{1}{\boldsymbol{P}^{\bar{H}}(R+1 \Delta R, Z) \boldsymbol{C}^{-1} \boldsymbol{P}(R+1 \Delta R, Z)} .
\end{aligned}
$$

The location of the source is given by the maximum of $S_{\mathrm{ML}}(R+1 \Delta R, Z)$, called here as parameter spectrum. This is also known as ambiguity surface, e.g., in [2].

\section{FAST MFP ALGORITHM}

In the proposed method, the computation of MFP output at different trial locations is modeled as a Fourier transform problem and the FFT is used to speed up the processing. This method is applicable to both horizontal and vertical arrays.

The acoustic pressure in a lossless waveguide at a sensor $i$, which is at depth $Z_{i}$, due to a trial source at location $(R+1 \Delta R, Z)$ is given by $[9$, p. 91]

$$
P\left(R+1 \Delta R, Z, Z_{i}\right)=\sum_{k=1}^{M} \frac{U_{k}(Z) U_{k}\left(Z_{i}\right) e^{j \xi_{k}(R+1 \Delta R)}}{\sqrt{\xi_{k}(R+1 \Delta R)}}
$$

where $\xi_{k}$ and $U_{k}$ are the $k$ th mode horizontal wave number and eigenfunction function, respectively, and $M$ stands for number of modes. The replica field vector at a vertical array due to a source at $(R+1 \Delta R, Z)$ can be written as

$$
\boldsymbol{P}(R+1 \Delta R, Z)=\frac{\boldsymbol{U} \boldsymbol{E}}{\sqrt{R+1 \Delta R}}
$$

where $U$ is a $L \times M$ matrix whose $(i k)^{\text {th }}$ element is given by $U_{i k}=\frac{U_{k}(z) U_{k}\left(z_{i}\right) e^{j \xi_{k} R}}{\sqrt{\xi_{k}}}$ $i=1,2, \cdots, L$

$$
k=1,2, \cdots, M
$$

where $L$ is the number of sensors, $M$ is the number of propagating modes in the channel, and

$$
\boldsymbol{E}=\left[e^{j \xi_{1} 1 \Delta R}, \cdots, e^{j \xi_{M} 1 \Delta R}\right]^{T} .
$$

Substituting the above expression for the replica field vector in the definition of the ML processor (1), we get

$$
S_{M L}(R+1 \Delta R, Z)=\frac{1}{E^{H} C^{\prime} E}
$$

where $\boldsymbol{C}^{\prime}=\boldsymbol{U}^{H} \boldsymbol{C}^{-1} \boldsymbol{U}$ and $\boldsymbol{C}$ is the covariance matrix of the received field. The above expression gives the processor output for a trial location $(R+1 \Delta R, Z)$. Since the strength of the normalized replica field vector does not depend on the trial source range (the normalization was done by dropping $\sqrt{R+1 \Delta R}$ from the denominator in (2)), the range factor has been removed from the above expression.

Next we shall show that the evaluation of above expression for the trial locations $(R, Z),(R+\Delta R, Z), \cdots,(R+$ $\left.L_{R} \Delta R, Z\right)$ is equivalent to finding the inverse Fourier transform of a sequence. Let

$$
G(R+1 \Delta R, Z)=\boldsymbol{E}^{H} \boldsymbol{C}^{\prime} \boldsymbol{E} \quad 1=0,1, \cdots, L_{R}
$$

where $L_{R}$ is the number of search points in range. Approximating the phase terms in $\boldsymbol{E}$ by integral multiples of $2 \pi / N$, assuming they are less than $2 \pi$, if not subtract integral multiples of $2 \pi$ and rearrange the vector $E$ such that the phase terms are in ascending order (corresponding changes will have to be done to $C^{\prime}$ also), we get

$$
\boldsymbol{E} \approx \tilde{\boldsymbol{E}}=\left[e^{j \frac{2 \pi}{N} n_{1} 1}, \cdots, e^{j \frac{2 \pi}{N} n_{M} 1}\right]^{T}
$$

where $N$, equal to 2 raised to an integer power, is a large integer greater than $L_{R}$ and integers $n_{i} \leq N$ for all $i$.

By introducing zero rows and zero columns into $C^{\prime}$ appropriately, $G(R+\Delta R, Z)$ can be written as

$$
\tilde{G}(R+1 \Delta R, z)=\tilde{\boldsymbol{E}}^{H} \boldsymbol{C}^{\prime} \tilde{\boldsymbol{E}}=\boldsymbol{E}^{\prime H} \boldsymbol{D} \boldsymbol{E}^{\prime}
$$

where

$$
\boldsymbol{E}^{\prime}=\left[1, e^{j \frac{2 \pi}{N} 1}, \cdots, e^{j \frac{2 \pi}{N}(N-1) 1}\right]^{T}
$$

and $\boldsymbol{D}$ is a new $N * N$ matrix obtained from $\boldsymbol{C}^{\prime}$ by introducing zero rows and zero columns so that the hermitian form in (6) remains unchanged. Consider an example:

$$
\begin{aligned}
\boldsymbol{e}^{T} \boldsymbol{C} \boldsymbol{e} & =\left[\begin{array}{l}
e_{1} \\
e_{2} \\
e_{3}
\end{array}\right]^{T}\left[\begin{array}{lll}
c_{11} & c_{12} & c_{13} \\
c_{21} & c_{22} & c_{23} \\
c_{31} & c_{32} & c_{33}
\end{array}\right]\left[\begin{array}{l}
e_{1} \\
e_{2} \\
e_{3}
\end{array}\right] \\
& =\left[\begin{array}{l}
e_{1} \\
a \\
e_{2} \\
e_{3}
\end{array}\right]^{T}\left[\begin{array}{llll}
c_{11} & 0 & c_{12} & c_{13} \\
0 & 0 & 0 & 0 \\
c_{21} & 0 & c_{22} & c_{23} \\
c_{31} & 0 & c_{32} & c_{33}
\end{array}\right]\left[\begin{array}{l}
e_{1} \\
a \\
e_{2} \\
e_{3}
\end{array}\right] .
\end{aligned}
$$

By actual evaluation of the above hermitian forms it can be seen that the value remains independent of the term " $a$ " introduced in the vector $e$ if we introduce a zero row and a zero column as shown. Like $\boldsymbol{C}$, the new matrix $\boldsymbol{D}$ is also Hermitian. Writing (6) in full form we have

$$
\tilde{G}(R+1 \Delta R, z)==\sum_{i=1}^{N} \sum_{m=1}^{N} D_{i j} e^{j \frac{2 \pi}{N}(m-i) 1} .
$$

For a Hermitian matrix $D$, performing the summation along the diagonals of the matrix, it can be shown [10], [11] that

$$
\tilde{G}(R+1 \Delta R)=2 \operatorname{Re}\left\{\sum_{k=0}^{N-1} F(k) e^{j \frac{2 \pi}{N} k 1}\right\}
$$


where $F(k)$ is expressible as a sum of diagonal elements of $\boldsymbol{D}$. For example, $F(0)$ is the sum of the main diagonal elements divided by two, $F(1)$ is the sum of the elements of the first diagonal above the main diagonal, $F(2)$ is equal to the sum of second main diagonal elements, and so on.

Thus with a single call to the FFT routine, the present algorithm is able to compute the parameter spectrum at all locations, namely, $(R, Z),(R+\Delta R, Z), \cdots,\left(R+L_{R} \Delta R, Z\right)$, for a fixed depth. By changing the value of $R$ and $Z$, and repeatedly applying this algorithm the entire region of interest can be scanned.

The above development is also valid for a horizontal array with a small change, namely in the computation of replica vector. Let $S_{i}$ be the distance of $i$ th sensor from the reference sensor (sensor $\{\#\} 1$, see Fig. (1(b)). Then the acoustic pressure at the $i$ th sensor due to a trial source at a distance $R+1 \Delta R$ from the reference sensor and depth $Z$ is given by

$$
\begin{aligned}
& P\left(R+1 \Delta R+S_{i}, Z, Z_{s}\right) \\
& =\sum_{k=1}^{M} \frac{U_{k}(Z) U_{K}\left(Z_{s}\right) e^{j \xi_{k}\left(R+1 \Delta R+S_{i}\right)}}{\sqrt{\xi_{k}\left(R+1 \Delta R+S_{i}\right)}} \\
& \approx \sum_{k=1}^{M} \frac{U_{k}(Z) U_{k}\left(Z_{s}\right) e^{j \xi_{k}\left(R+1 \Delta R+s_{i}\right)}}{\sqrt{\xi_{k}(R+1 \Delta R)}}
\end{aligned}
$$

assuming $S_{i} \ll(R+1 \Delta R)$. The replica field vector at the horizontal array can be written as

$$
\boldsymbol{P}(R+1 \Delta R, Z) \approx \frac{\boldsymbol{U E}}{\sqrt{R+1 \Delta \bar{R}}}
$$

where $\boldsymbol{U}$ is a matrix whose $(i, k)$ th element is given by

$$
U_{i k}=\frac{U_{k}(z) U_{k}\left(z_{s}\right) e^{j \xi_{k}\left(R+S_{i}\right)}}{\sqrt{\xi_{k}}} .
$$

It may be noted that the above algorithm will work for range localization and not for depth localization described in [12]. However, two-dimensional scan is possible by repeated application of fast MFP.

\section{PRocessing SPEed}

In the proposed fast MFP, computation of the processor output for a set of trial source locations is modeled as a Fourier transform problem. Hence, we expect an improvement in the processing speed inherent in all FFT-based processing schemes. The ratio of the required number of complex multiplications in the conventional method, i.e., computing the replica field for each trial location and then computing the MRP output, to that in fast MFP is given by

Speed Gain $\approx \frac{\left(L^{2}+3 L M+2 L\right) L_{R}}{3 L M+L M^{2}+L^{2} M+x L_{R} \log _{2} x L_{R}}$

where $x=N / L_{R}$ is the ratio of the total number of IDFT coefficients computed to the number of IDFT coefficients actually used by this algorithm. Here, only the basic operations are taken into account. In the numerator, a minimum of 3LM to compute the replica field vector, $\left(L^{2}+L\right) L_{R}$ to evaluate the hermitian form $\boldsymbol{P}^{H} \boldsymbol{C}^{-1} \boldsymbol{P}$, and $L L_{R}$ to normalize the replica

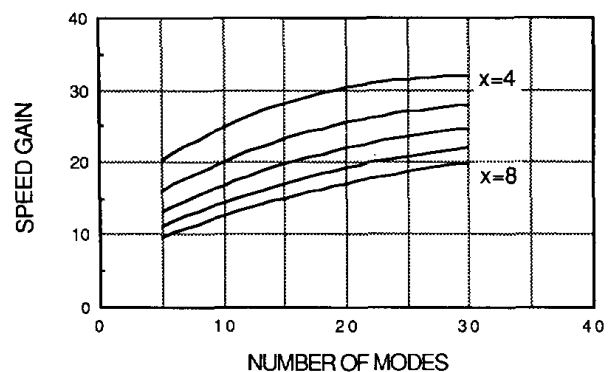

Fig. 2. Speed gain as a function of number of modes $\left(L_{R}=1000\right)$ for channel shown in Fig. 1.

field. These estimates are based on [2, equations (3), (5), and (22)]. Based on these equations a computer program was written for evaluating the parameter spectrum. Some saving in the number of operations can be made by exploiting the structure of matrix $\boldsymbol{P}$ in (2) as a product of two matrices $\boldsymbol{U}$ and $\boldsymbol{E}$, of which $\boldsymbol{U}$ is a function of depth alone (and fixed range); hence it need be evaluated only once. In the denominator, $M^{2} L+L^{2} M$ to evaluate $\boldsymbol{U}^{H} \boldsymbol{C}^{-1} \boldsymbol{U}, x L_{R} \log _{2} x L_{R}$ for the FFT, and $3 L M$ to form the matrix $\boldsymbol{U}$. Since the normalization was done by simply dropping the denominator term in (2) no computation was involved.

The expected gain in the processing speed is plotted in Fig. (2) as a function of the number of propagating modes, $M$, in the channel for different values of $x\left(L_{R}=1000\right)$. The figure shows that the expected gain in the processing speed increases with the complexity of the field structure and decreases with increasing value of $x$. The physical significance of the value of $x$ will be made clear in the next section. Note that the localization accuracy depends on the number of modes and hence, the gain is related to the performance of the MFP.

A numerical simulation was carried out to confirm the predicted improvement in the processing speed. For the purpose of simulation we have assumed $N=4096, S N R=0 \mathrm{~dB}$, $L_{R}=1000$, and $\Delta R=10 \mathrm{~m}$. The processing speed gain was measured by noting the cpu time required on Microvax II (work station) by the conventional MFP and that required by the fast MFP. The ratio of the CPU times is shown in Fig. 3 along with the predicted result. The simulation results generally agree with the predicted ones. The observed differences are probably due to unaccounted operations in the actual simulation. The gain in processing speed is generally large when the channel is rich in modes, that is, for large $M$.

Note that this algorithm uses only the first $L_{R}$ IDFT coefficients out of $N$ computed coefficients. Since $L_{R} \ll N$ for low quantization error, one can make use of a more efficient FFT algorithm [13] to further improve upon the gain in the processing speed. The algorithm does not require any extra storage space since only nonzero elements of $\boldsymbol{D}$ and their locations need to be stored.

\section{EFFECT OF QUANTIZATION ERROR}

In the development of fast MFP an approximation was made in (5), namely,

$$
\xi_{i} \Delta R=\frac{2 \pi}{N} n_{i}+\epsilon_{i}
$$




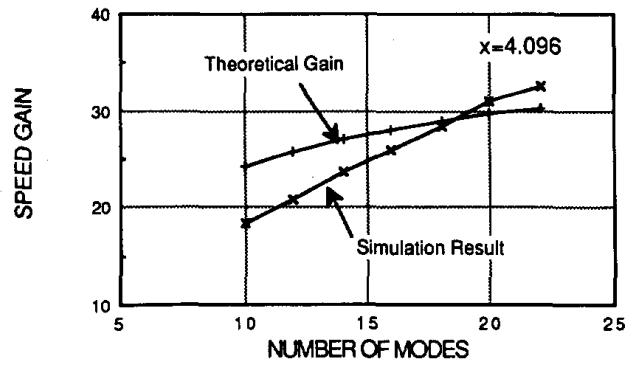

Fig. 3. Simulation results.

where $\left|\epsilon_{i}\right| \leq \pi / N$ is the quantization error. For an arbitrary value of 1 the quantization error may be approximated by

$$
\begin{aligned}
\xi_{i} 1 \Delta R= & \frac{2 \pi}{N} 1 n_{i}+\epsilon_{i l} \quad l=0,1,2, \cdots, L_{R} \\
& \times\left|\epsilon_{i l}\right| \leq l \frac{\pi}{N} .
\end{aligned}
$$

From (11) we note that as 1 increases the quantization error also increases. To keep the quantization error small the maximum value of 1 , namely $L_{R}$, is chosen to be much smaller than $N$. The quantization error introduces a random phase in the expression for the replica field (2). Since in MFP the replica field vector for different trial locations are matched with the received field, fast MFP output is expected to be sensitive to this quantization error.

The effect of phase quantization on the fast MFP output (rather on the inverse of the output) is derived below. In (6) we introduce the phase quantization error as modeled in (11)

$$
\tilde{G}(R+1 \Delta R, Z)=\tilde{E}^{H} C^{\prime} \tilde{E}
$$

where the quantization error in $\tilde{E}$ is explicitly shown,

$$
\tilde{\boldsymbol{E}}=\left[e^{j\left(\xi_{1} 1 \Delta R-\epsilon_{11}\right)}, \cdots, e^{j\left(\xi_{M} 1 \Delta R-\epsilon_{M 1}\right)}\right]
$$

On expanding the hermitian form we obtain

$$
\tilde{G}(R+1 \Delta R, Z)=\sum_{i=1}^{M} C_{i i}^{\prime}+\sum_{\substack{i=1 \\ i \neq k}}^{M} \sum_{k=1}^{M} C_{i k}^{\prime} a *_{i} a_{k}
$$

where $a_{i}=e^{j\left(\xi_{i} 1 \Delta R-\epsilon_{i 1}\right)}$. Now we model $\epsilon_{i 1}$ as a random variable which is uniformly distributed over a range, $\pm 1(\pi / N)$. Taking the expected operation on both sides of (13) we obtain

$$
\begin{aligned}
& E\{\tilde{G}(R+1 \Delta R, Z)\} \\
& =\sum_{i=1}^{M} C_{i i}^{\prime}+\eta^{2} \sum_{\substack{i=1 \\
i \neq k}}^{M} \sum_{\substack{k=1 \\
i \neq k}}^{M} C_{i k}^{\prime} e^{j\left(\xi_{k}-\xi_{i}\right) 1 \Delta R} \\
& =\left(1-\eta^{2}\right) \sum_{i=1}^{M} C_{i i}^{\prime}+\eta^{2} G(R+1 \Delta R, z)
\end{aligned}
$$

where $\eta=(\sin (1(\pi / N)) /(1(\pi / N)))$. The first term on the right-hand side of (14) is the error term caused by phase
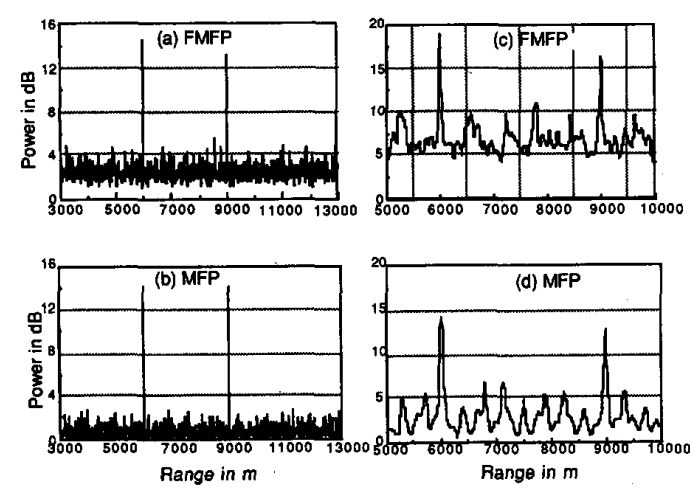

Fig. 4. Comparison of fast MFP (FMFP) and conventional MFP for two different channels. (a) and (b) For hard bottom channel as shown in Fig. 1. (c) and (d) For soft bottom, uniform sound speed (Pekeris) channel.

quantization. For $1 \ll N$, that is, $L_{R} \ll N$ the error term will be very small.

To study the effect of the quantization error on the fast MFP output, the several simulations were performed. Two uncorrelated sources $(\mathrm{SNR}=0.0 \mathrm{~dB})$ at same depth $(50 \mathrm{~m})$ but at $6 \mathrm{~km}$ and $9 \mathrm{~km}$ range, respectively, were assumed. Other parameters of the channel are as in Fig. 1. We have applied both MFP and fast MFP to same covariance matrix (assumed to be error free). Fig. 4(a) shows the fast MFP output and Fig. 4(b) shows the MFP output. The source peaks are correctly located. The source at $9 \mathrm{~km}$ appears to be attenuated (about $2 \mathrm{~dB}$ ) on account of quantization errors. However, the sidelobe level is noticeably higher in the fast MFP output. We have also considered a Pekeris channel (sound speed in water $=1500 \mathrm{~m} / \mathrm{s}$ and in sediment $1620 \mathrm{~m} / \mathrm{s}$ and density of water $=1 \mathrm{gm} / \mathrm{cc}$ and of sediment $=1.77 \mathrm{gm} / \mathrm{cc}$ ) with two sources at depth $50 \mathrm{~m}$ and range $6000 \mathrm{~m}$ and $9000 \mathrm{~m}$, respectively. The fast MFP output is shown in Fig. 4(c) and that of MFP in Fig. 4(d). The sources are correctly located but the character of the background is now totally different.

Further simulations were carried out to clearly bring out the effects of the quantization error on the source peak height. A source was successively placed at $(3000,50),(3020,50)$ $(3040,50)$, and so on. The power received at the array was normalized for each source position so that the source peak is expected to be same for all source locations. The fast MFP was used to compute the source peak and the largest sidelobe for each position of the source. The results are shown in Fig. 5(a) for 0.0-dB SNR and in Fig. 5(b) for 10-dB SNR. We note that the source peak height decreased with increasing souce range, but the largest sidelobe level remained practically unchanged. This pehnomenon was further studied in another simulation using the same channel (Fig. 1) but with positive sound speed gradient, $1500 \mathrm{~m} / \mathrm{s}$ to $1510 \mathrm{~m} / \mathrm{s}$. We have found a similar behavior. From this one may conclude that the effect of the quantization error is to reduce the source peak level; the reduction depends on the value of 1 at the true source position.

From (14) we note that the inverse of the source peak in ML spectrum is linearly dependent on $\{\eta\}^{2}$. The verification of this prediction is shown in Fig. 6. A least-squares fit is also 


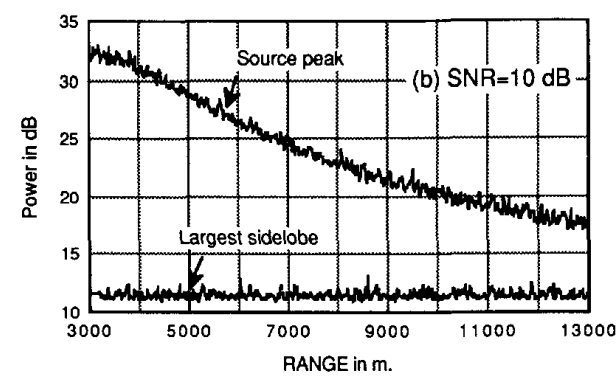

Fig. 5. The effect of quantization on the source peak height as a function $I \Delta R$ for the channel shown in Fig. 1 . SNR $=0.00 \mathrm{~dB}$ in (a) and $10 \mathrm{~dB}$ in (b). The height of the largest sidelobe observed in the range $\pm 2500 \mathrm{~m}$ from the true source position is found to be unaffected by the quantization error.

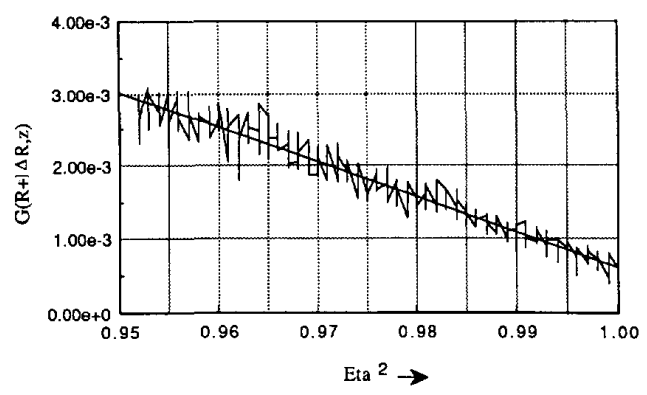

Fig. 6. Inverse of source peak height is plotted against $\eta^{2}$ defined in (14). The quantization noise model developed here predicts a linear relation (14) between the inverse of source peak and $\eta^{2}$. This prediction is verified in the above figure. A least-squares linear fit is also shown in the figure.

shown. Finally, we observe that the reduction of peak height appears to be greater at higher SNR $(10 \mathrm{~dB})$ than at lower SNR (0.0). Such a dependence of sensitivity on the SNR was also noted by Gingras [6] for any mismatch between replica field and received field.

The choice of $x\left(=N / L_{R}\right)$ is based on the amount of reduction in the source peak height in relation to sidelobe height one is prepared to accept, keeping in mind the fact that the speed gain depends on the value of $x$. In practical applications, this algorithm should be applied in two steps. First, apply this algorithm for a reasonably large value for $x$ to estimate the probable source locations. In the second step, apply the algorithm selectively near each probable source location, to confirm the presence of a source and to estimate its power, taking an appropriate value of $R$ such that the value of 1 at each potential source location is so small that the algorithm will give a reasonably accurate estimate of the source peak.

\section{CONCLUSION}

A fast algorithm based on FFT has been developed to implement the matched field algorithm (MFP) for localization (range) of a sound source in a shallow water channel. We have shown that the processing speed can be considerably improved (20 to 30 times) at the expense of a slight reduction of the source peak height. We have demonstrated both theoretically and through numerical experiments that the effect of quantization is largely to reduce the source peak height, depending upon the size of DFT.

\section{REFERENCES}

[1] H. P. Bucker "Use of calculated sound fields and matched field detection to locate sound sources in shallow water," J. Acoust. Soc. Am., vol. 59, pp. 368-373, Feb. 1976.

[2] R. G. Fizell, "Application of high resolution processing to range and depth estimation using ambiguity surface," J. Acoust. Soc. Am., vol. 82, pp. 606-613, Aug. 1987.

[3] A. B. Baggeroer, W. A. Kuperman, and H. Schmidt, "Matched field processing: Source localization in correlated noise as a parameter estimation problem," J. Acoust. Soc. Am., vol. 83, pp. 571-587, Feb. 1988.

[4] J. M. Ozard, "Matched field processing in shallow water for range, depth and bearing determination: Results of experiment and simulation," $J$. Acoust. Soc. Am., vol. 86, pp. 744-753, Aug. 1989.

[5] R. M. Hamson and R. M. Heitmeyer, "Environmental and system effects on source localization in shallow water by MFP of a vertical array," $J$. Acoust. Soc. Am., vol. 86, pp. 1950-1959, Nov. 1989.

[6] D. F. Gingras, "Methods for predicting the sensitivity of MFP to mismatch," J. Acoust. Soc. Am., vol. 86, pp. 1940-1949, Nov. 1989.

[7] J. Capon, "High resolution frequency-wavenumber spectrum analysis," Proc. IEEE, vol. 57, pp. 1408-1418, 1969.

[8] J. M. Ozard, P. Zakarauskas, and P. W. Ko, "Matched field Processing using a neural network with preprocessing," J. Acoust. Soc. Am. Suppl. 1, vol. 88, Fall 1990.

[9] L. Brekhovskikh and Y. Lysanov, Fundamentals of Ocean Acoustics. New York: Springer-Verlag, 1982.

[10] A. M. Musicus, "Fast MLM power spectrum estimation from uniformly spaced correlation," IEEE Trans. Acoust. Speech, Signal Processing, vol. 33, 1985, pp. 1333-1335.

[11] A. C. Monteiro, "Estimating the bearing of a moving source," M.E dissertation, Dept. Elect. Comm., Eng. IISc, 1988.

[12] T. C. Yang, "Modal shading coefficients for high resolution source depth localization," J. Acoust. Soc. Am., vol. 87, pp. 668-672, Feb. 1990.

[13] J. D. Markel, "FFT pruning," IEEE Trans. Audio Electro. Acous., vol AU-19, pp. 305-311, Dec. 1971

S. Aravindan, photograph and biography not available at time of publication.

N. Ramachandran, photograph and biography not available at time of publication.

Prabhakar S. Naidu, photograph and biography not available at time of publication. 